\title{
TEORÍAS Y MÉTODOS EN LA INVESTIGACIÓN SOBRE TURISMO, GÉNERO Y MUJERES EN IBEROAMÉRICA: UN ANÁLISIS BIBLIOGRÁFICO
}

\author{
Laura Paola Vizcaíno Suárez* \\ Rocío Serrano Barquín* \\ Graciela Cruz Jiménez* \\ Universidad Autónoma del Estado de México \\ María José Pastor Alfonso** \\ Universidad de Alicante
}

\section{RESUMEN}

Este artículo realiza una revisión integradora de la investigación sobre turismo, género y mujeres, publicada en 11 revistas indizadas de turismo en idioma español y portugués desde su primer año de divulgación. Se estableció como requisito que al menos una de las autoras o autores tuviera adscripción en una institución iberoamericana. Se siguió la propuesta metodológica de Henderson y Gibson (2013) con la finalidad de centrar el análisis en las relaciones de género y las experiencias de las mujeres en el turismo. Se seleccionaron 31 artículos completos para análisis de contenido. El estudio identifica y analiza las temáticas de la investigación sobre turismo, género y mujeres en la región iberoamericana, los conceptos más relevantes, los métodos e instrumentos de análisis, así como los principales enfoques teóricos.

Palabras clave: investigación; turismo; género; mujeres; Iberoamérica.

Recibido: 23 de febrero de 2015

Devuelto para su revisión: 23 de septiembre de 2015

Aceptado: 20 de noviembre de 2015

* Centro de Investigación y Estudios Turísticos. Facultad de Turismo y Gastronomía. Universidad Autónoma del Estado de México. Cerro de Coatepec S/N. Ciudad Universitaria. C.P. 50110. TOLUCA, Estado de México (México).E-mail: paola.vizcaino.suarez@gmail.com,rocioserba14@gmail.com,gracicj@hotmail.com

** Departamento de Humanidades Contemporáneas. Universidad de Alicante. Apartado 99. 03080 ALICANTE (España).E-mail: josefa.pastor@ua.es 


\title{
Theories and methods in research on tourism gender and women in Ibero-america: a bibliographic analysis
}

\begin{abstract}
This article conducts an integrative review of research on tourism, gender and women, published in 11 major tourism refereed journals in Spanish and Portuguese from the first year of publication of each journal. The review established that at least one of the authors was ascribed to an Ibero-American institution. We followed the methodological proposal of Henderson and Gibson (2013) in order to focus the analysis on gender relations and women's experiences in tourism. Thirty one complete papers were selected for content analysis. This study identifies and analyzes the main themes on the tourism, gender and women research in the Ibero-American region, as well as relevant concepts, methods, instruments and theoretical approaches.
\end{abstract}

Keywords: research; tourism; gender; women; Ibero-America.

\section{INTRODUCCIÓN}

Este artículo tiene el propósito de plasmar los resultados de una revisión integradora sobre la investigación en turismo, género y mujeres en Iberoamérica. Para ello, se examinaron 31 artículos completos publicados en 11 revistas indizadas de turismo de la región desde su primer año de publicación. Su contenido forma parte de la investigación «Turismo y procesos de empoderamiento de las mujeres artesanas de Metepec, México», que se desarrolla en el programa de Doctorado en Estudios Turísticos de la Facultad de Turismo de la Universidad Autónoma del Estado de México, en la línea de investigación sobre turismo, desarrollo local y sustentabilidad.

A partir de la exploración con dichos fines, se identifica que durante años, la investigación turística priorizó el análisis de las dimensiones económico-administrativas del turismo, soslayando las dimensiones políticas y sociales, donde el género juega un papel primordial al articular, junto con otras categorías estratificadoras como la clase y el origen étnico, las cuestiones de poder, control y equidad en los procesos y las prácticas turísticas (Enloe, 1989; Kinnaird y Hall, 1994; Swain, 1995). Desde mediados de 1990, el giro crítico en los estudios turísticos ha mostrado un mayor interés en el análisis de las dimensiones políticas y sociales del turismo, incluyendo el análisis de las relaciones de género (Pritchard, Morgan y Ateljevic, 2011).

Para algunas autoras, la ausencia del género, así como la marginalización de las mujeres en los estudios turísticos, no es tan sorprendente, dado que la investigación social en general aparece dominada por una perspectiva tradicional de investigación, basada en la universalización de las experiencias, las percepciones y las creencias masculinas (Harding, 1986; Stanley y Wise, 1993). Henderson (1994) estableció cinco fases en la evolución del análisis de género en los estudios de ocio, que fueron retomadas por Swain (1995) para caracterizar también a los estudios turísticos: 1) invisible (sin mujeres); 2) compensatorio («añada mujeres y agite»); 3) diferencias dicotómicas (diferencias sexuales); 4) perspec- 
tiva feminista (centrada en las mujeres); y 5) marcos conscientes del género (análisis de las relaciones, roles y estereotipos que sitúan a las mujeres en una posición de subordinación frente a los varones).

Los análisis de género en el turismo pueden centrarse en uno o ambos sexos (Henderson, 1994). Su objetivo es teorizar por una parte, sobre cómo los comportamientos y los roles responden a construcciones sociales de género; por otra, abordar cómo la división sexual del trabajo refleja diferencias y la manera en que las estructuras sociales incorporan valores y otorgan ventajas a través de relaciones jerárquicas (Swain, 1995).

Para el análisis de las dimensiones de género en el turismo, Kinnaird y Hall (1996) propusieron un «marco consciente del género» con base en las siguientes premisas conceptuales: a) Las actividades y los procesos relacionados con el desarrollo turístico se construyen en sociedades con relaciones de género; b) las relaciones de género se alimentan de y re-alimentan las prácticas políticas, económicas, sociales y culturales donde se desarrolla el turismo; c) las discusiones y las relaciones de género en el turismo, se relacionan con cuestiones de poder, control y equidad.

Actualmente, se observan tres grandes áreas de estudio en la investigación sobre turismo y género en la literatura internacional (Gibson, 2001), que se identificaron también en la iberoamericana:

1. Estudios centrados en las y los turistas (consumidoras y consumidores), donde se analizan diferencias de género en el consumo turístico y el comportamiento en los viajes, aunque no siempre se explican dichas diferencias.

2. Estudios centrados en las y los anfitriones del turismo (productoras y productores). Se analizan las diferencias de género en la producción turística; las oportunidades en el empleo turístico para mujeres y varones; la segregación laboral y la discriminación en el empleo. Las más de las veces se analiza el rol de las mujeres como empleadas en el sector turístico y no como empleadoras (Peeterson y Ateljevic, 2009).

3. El turismo como parte de las construcciones sociales de género, con referencia a los objetos e imágenes empleados en la promoción turística, que se caracterizan como masculinos o femeninos.

En la literatura anglosajona se han revisado las dimensiones de género y la participación de las mujeres en el ocio (Henderson 1996; Henderson y Hickerson, 2007; Henderson y Gibson, 2013) y en el turismo (Swain, 1995; Gibson, 2001). En contraste, en la producción científica iberoamericana existe un vacío sobre las teorías y métodos en la investigación sobre turismo, género y mujeres. Por tanto, este artículo contribuye a cubrir dicho vacío, a la vez que aporta un primer diagnóstico sobre el estado del arte de la investigación sobre turismo, género y mujeres, con la finalidad de promover y facilitar debates teóricos, conceptuales y metodológicos para avanzar esta línea de investigación. Como estrategia para analizar la literatura y descubrir relaciones generales entre conceptos, teorías y métodos en las investigaciones, se empleó la revisión integradora. Ésta también permite realizar inferencias sobre patrones y tendencias con relación a las temáticas que se abordan en la literatura (Cooper, 1982). 


\section{METODOLOGÍA}

Tras observar una escasez de estudios bibliográficos que examinen la temática de turismo, género y mujeres en al ámbito iberoamericano y dado que, en general, no existe consenso sobre la clasificación de las revistas indizadas en turismo (Zhao y Ritchie, 2007), se llevó a cabo un análisis de las publicaciones en esta región. A pesar de que hay un cierto grado de subjetividad en la selección de las publicaciones para la revisión integradora, se tomaron en cuenta las recomendaciones de Zhao y Ritchie (2007) con relación a: a) factor de impacto de las revistas; b) reconocimiento internacional (o reconocimiento en la región de estudio); c) trayectoria de publicación; y d) posibilidad de incluir un amplio rango de perspectivas sobre la investigación turística. Con base en las recomendaciones anteriores, se seleccionaron 17 revistas indizadas de la región iberoamericana: 3 latinoamericanas en idioma español, 5 brasileñas, 7 españolas y 2 portuguesas. La muestra de estudio incluyó a las publicaciones que arrojaron resultados con uno o más de los términos de búsqueda y que permitieron el acceso a los textos completos. Se identificaron artículos relevantes en 11 de las 17 revistas indizadas, de las cuales 9 se publican en idioma español y 2 en portugués. El período de estudio abarcó el año inicial de publicación de cada revista a la fecha, lo que arrojó artículos de 1998 a 2014.

Las preguntas de investigación para esta revisión se relacionan principalmente con las siguientes variables: temática de estudio en los artículos seleccionados; los conceptos más relevantes, los métodos e instrumentos de análisis y los principales enfoques teóricos. Debido a que el propósito de esta revisión integradora fue ampliar el conocimiento sobre las relaciones de género y las experiencias de las mujeres en el turismo, se apegó a la propuesta de Henderson y Gibson (2013) y se examinó el contenido de los artículos utilizando las palabras clave: mujer(es), niña(s), género, empoderamiento, feminismo; o palabras relacionadas como familia(s), perspectiva de género, teoría feminista que aparecieran en el título, el resumen o entre las palabras clave identificadas. No se incluyeron los artículos centrados en cuestiones administrativas, recursos humanos o marketing, salvo que incorporaran una definición de género o alguna referencia teórico-metodológica de la perspectiva de género. La revisión tampoco incluye ponencias de congresos ni capítulos de libros. Se centra exclusivamente en artículos de revistas indizadas porque son de más fácil acceso a través de los recursos electrónicos (índices como Google Scholar y Scopus o sitios Web de las revistas). Se estableció como requisito que al menos una de las autoras o autores tuviera adscripción a una institución iberoamericana. La muestra de revistas que arrojaron resultados y el número de artículos seleccionados para análisis, aparecen en la Tabla 1.

Un total de 31 artículos de las revistas mencionadas en la Tabla 1 cumplieron los criterios establecidos, por lo que se procedió a su revisión, síntesis y análisis. Los resultados se analizaron mediante técnicas de descripción cuantitativa (características tanto de las investigaciones, como de las investigadoras e investigadores), así como técnicas cualitativas de análisis y comparación de contenido de los artículos seleccionados. Se identificaron, analizaron y categorizaron todos los artículos de acuerdo a las características de las investigaciones. Después, se revisaron las notas preliminares y se llevó a cabo una discusión sobre los patrones que emergieron con respecto a las temáticas de investigación, la especificidad de los conceptos de género en los objetivos o preguntas de investigación 
Tabla 1

\section{MUESTRA DE REVISTAS ANALIZADAS}

\begin{tabular}{|l|c|c|c|}
\hline \multicolumn{1}{|c|}{ Revistas } & $\begin{array}{c}\text { País de } \\
\text { publicación }\end{array}$ & $\begin{array}{c}\text { Idiomas de } \\
\text { publicación* }\end{array}$ & $\begin{array}{c}\text { Número de } \\
\text { artículos } \\
\text { sobre género y } \\
\text { mujeres }\end{array}$ \\
\hline El Periplo Sustentable & México & E, P & 1 \\
\hline Estudios y Perspectivas en Turismo & Argentina & E & 5 \\
\hline $\begin{array}{l}\text { Revista Brasileira de Pesquisa em } \\
\text { Turismo }\end{array}$ & Brasil & P, E, I & 3 \\
\hline Análisis Turístico & España & E & 2 \\
\hline Cuadernos de Turismo & España & E, I & 6 \\
\hline Estudios Turísticos & España & E & 1 \\
\hline Investigaciones turísticas & España & E, P, V & 1 \\
\hline PASOS & España & E, P, I, F & 8 \\
\hline ROTUR & España & E, I, P & 2 \\
\hline TURyDES & España & E, P & 1 \\
\hline $\begin{array}{l}\text { Encontros Científicos }- \text { Tourism \& } \\
\text { Management Studies }\end{array}$ & Portugal & P, E, I & 1 \\
\hline \multicolumn{1}{|c|}{ TOTAL: 11 revistas } & & & 31 artículos \\
\hline
\end{tabular}

Fuente: elaboración propia a partir de los resultados de búsqueda.

*E-español, F-francés, I-inglés, P-portugués, V-valenciano

y las principales teorías y métodos que sustentan los estudios. El análisis de contenido permitió comparar y contrastar el propósito y los resultados de los artículos. En la sección de discusión se ofrece una síntesis e interpretación de los aspectos teórico-metodológicos de los artículos analizados, así como de su autoría.

\section{ANÁLISIS DE LAS INVESTIGACIONES}

\subsection{Aspectos generales}

En este apartado se presentan los resultados del análisis de los 31 artículos abordados mediante técnicas de descripción cuantitativa para identificar aspectos generales de las investigaciones (autoría, año de publicación, idioma), así como de las investigadoras e investigadores (género, área de adscripción académica, universidad y país), con la finalidad de identificar a las personas e instituciones que empiezan a desarrollar la línea de turismo y género en la región estudiada. En la Figura 1 se muestra la distribución de los artículos analizados por año de publicación. 


\section{Figura 1 \\ NÚMERO DE ARTÍCULOS PUBLICADOS POR AÑO DE LA MUESTRA}

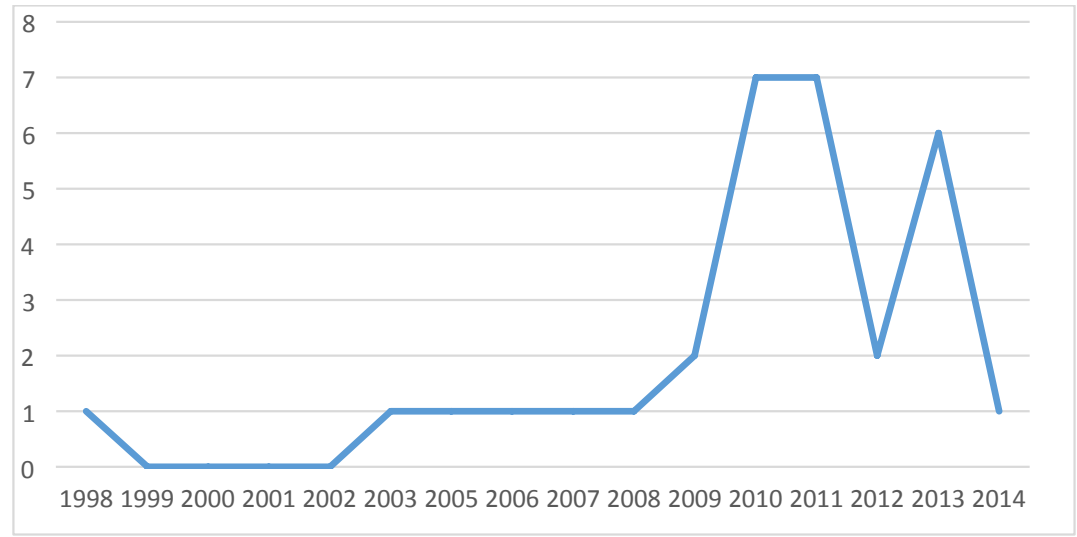

Fuente: elaboración propia con base en la muestra de artículos analizados.

Con base en los criterios de búsqueda, se detectó un solo artículo publicado en 1998 y ninguno entre 1999 y 2002. La búsqueda arrojó resultados nuevamente a partir de 2003 a razón de un artículo por año en la publicación global de las 11 revistas durante el período de 2003 a 2008. Se observa una tendencia al alza a partir de 2009, llegando a un máximo de siete documentos en 2010 y 2011. En general, predominan los artículos en español (28) sobre los publicados en portugués (2) o inglés (1), debido a que la muestra concentra un mayor número de revistas originarias de España y de países latinoamericanos de habla hispana. Con respecto a la autoría, del total de 52 autoras y autores, el $81 \%$ son mujeres (41) y el $19 \%$ restante hombres (10); éstos en su mayoría aparecen como coautores y no como autores únicos, salvo en un caso. Esta tendencia es consistente con las revisiones de la literatura que se han hecho en el contexto anglosajón sobre dimensiones de género y mujeres en el ocio (Henderson y Gibson, 2013) y en el turismo (Swain, 2005), donde la mayoría de los artículos publicados son de autoría femenina.

Las investigadoras y los investigadores de la muestra laboran en 24 universidades o centros de investigación de siete países de la región iberoamericana (Argentina, Brasil, España, México, Perú, Venezuela y Portugal), como se aprecia en la Tabla 2.

Con respecto al departamento de adscripción académica, poco más de un tercio de la muestra de investigadoras e investigadores pertenece a Facultades, Escuelas o Centros de investigación especializados en Turismo (32.7\%) y un cuarto corresponde a las Áreas o Departamentos económico-administrativos (25\%). El resto de la muestra se distribuye entre las áreas de: Ingeniería, Arquitectura o Diseño (9.6\%), Departamentos interdisciplinarios de desarrollo sustentable (9.6\%), Departamentos de Ciencias Humanas, Contemporáneas y Comunicación (7.8\%), Ciencias Sociales, Sociología o Antropología (5.8\%), Áreas especializadas en estudios de género (5.8\%) y Departamento de Geografía Humana (3.9\%). Lo anterior refleja la diversidad de enfoques a partir de los que se pueden abordar las interrelaciones y conexiones entre el fenómeno turístico y la categoría de género. 
Tabla 2

UNIVERSIDADES O CENTROS DE INVESTIGACIÓN POR PAÍS

\begin{tabular}{|c|}
\hline Argentina \\
\hline Centro de Investigaciones y Estudios Turísticos de Buenos Aires \\
\hline Brasil \\
\hline Fundación Getulio Vargas \\
\hline Universidad del Estado de Río de Janeiro \\
\hline Universidad Federal de Bahía \\
\hline Universidad Federal de Juis de Fora \\
\hline Universidad Federal de Río Grande del Sur \\
\hline México \\
\hline Colegio de Postgraduados \\
\hline Universidad Autónoma de Baja California \\
\hline Universidad Autónoma de Baja California Sur \\
\hline Universidad Autónoma del Estado de México \\
\hline Universidad del Mar \\
\hline Perú \\
\hline Pontifica Universidad Católica del Perú \\
\hline Venezuela \\
\hline Universidad de Oriente \\
\hline España \\
\hline Escuela Universitaria de Turismo de La Coruña \\
\hline Escuela Universitaria de Turismo de Málaga \\
\hline Universidad Autónoma de Madrid \\
\hline Universidad Complutense de Madrid \\
\hline Universidad de Alicante \\
\hline Universidad de Burgos \\
\hline Universidad de Valencia \\
\hline Universidad de Valladolid \\
\hline Universidad de Vigo \\
\hline Universidad de Zaragoza \\
\hline Portugal \\
\hline Instituto Universitario de Lisboa \\
\hline
\end{tabular}

Fuente: elaboración propia con base en la muestra de artículos analizados. 


\subsection{Métodos de análisis}

En las publicaciones se examinaron los métodos de análisis, los instrumentos de recolección de datos y las características de las poblaciones estudiadas. Se examinaron los métodos de análisis y se calculó el porcentaje de estudios que utilizaron cada uno de los métodos e instrumentos identificados, como se muestra en la Tabla 3.

\section{Tabla 3 \\ MÉTODOS E INSTRUMENTOS DE ANÁLISIS}

\begin{tabular}{|l|c|c|}
\hline \multicolumn{1}{|c|}{ Métodos e instrumentos de análisis } & $\begin{array}{c}\text { No de } \\
\text { artículos }\end{array}$ & $\begin{array}{c}\text { Porcentaje } \\
(\%)\end{array}$ \\
\hline $\begin{array}{l}\text { Cualitativo: entrevistas semi-estructuradas o en } \\
\text { profundidad, observación no participante y participante }\end{array}$ & 13 & $42 \%$ \\
\hline $\begin{array}{l}\text { Cuantitativo: análisis de bases de datos o fuentes } \\
\text { estadísticas, cuestionarios ad-hoc / encuestas }\end{array}$ & 7 & $23 \%$ \\
\hline $\begin{array}{l}\text { Métodos mixtos: encuestas cuantitativas y entrevistas } \\
\text { cualitativas / bloques de preguntas abiertas y cerradas }\end{array}$ & 7 & $23 \%$ \\
\hline Análisis histórico & 2 & $6 \%$ \\
\hline Análisis conceptual & 2 & $6 \%$ \\
\hline
\end{tabular}

Fuente: elaboración propia con base en la muestra de artículos analizados.

En las investigaciones analizadas sobre turismo, género y mujeres, predomina el uso de métodos cualitativos (entrevistas semiestructuradas o en profundidad, observación participante y no participante), en concordancia con los objetivos de investigación, generalmente enfocados a analizar, comprender, conocer, estudiar, examinar, identificar o indagar sobre: a) las dinámicas y relaciones de género en el turismo, b) el papel de la mujer en el desarrollo turístico (como trabajadora formal o informal, directiva o empresaria), o bien c) los discursos y representaciones turísticas sobre las mujeres. En este sentido, la elección de métodos cualitativos resulta útil para «indagar a fondo» sobre las actividades, contribuciones, experiencias y/o prácticas de las mujeres en el turismo. Como señala una de las autoras: «es necesario conocer la forma en que (las mujeres) experimentan $s u$ participación; considerando como punto de partida que dicha experiencia se ve condicionada por las relaciones productivas, sociales y de género construidas a partir de parámetros sociales y heterogéneos en el medio rural» (Díaz Carrión, 2010: 157) o en cualquier otro medio donde se desenvuelvan.

Tras los métodos cualitativos, las investigaciones examinadas se decantan en igual proporción por métodos cuantitativos y mixtos. La elección de métodos cuantitativos (análisis de bases de datos o fuentes estadísticas, cuestionarios ad-hoc o encuestas) suele responder al objetivo de analizar las diferencias de género con relación a variables específicas, como pueden ser: género y nuevas tecnologías (Diéguez, Gueimonde y 
Blanco, 2011); género y éxito empresarial (Diéguez, Gueimonde y Sinde, 2010); género y preferencias de compra (Prodanova y San Martín, 2013); género y empleo turístico (Sarasa, 1998); género y desigualdad laboral (Sigüenza, Brotons y Huete, 2013); entre otras. Por su parte, la elección de métodos mixtos aparece ligada a la necesidad de complementar los datos cuantitativos, que permiten situar la participación de las mujeres en los estudios, con datos cualitativos, que permiten aprehender las experiencias subjetivas o cuestiones simbólicas de dicha participación (ver por ejemplo, Iñiguez, Plumed y Latorre, 2013; o Tomio y Ullrich, 2011).

Los análisis históricos y los análisis conceptuales tienen menor representación en la muestra de estudios, pero no por ello dejan de ser importantes. Los primeros responden al interés de las autoras por mostrar los cambios en las estructuras sociales que han permitido la incorporación de las mujeres a campos tradicionalmente considerados masculinos (trabajo productivo, deportes, turismo). Los segundos son congruentes con los objetivos de avanzar en construcciones teóricas o conceptuales del turismo y la perspectiva de género.

Las poblaciones examinadas en los estudios empíricos $(n=25)$ varían con relación a la edad, origen étnico, nacionalidad, nivel educativo y capacidades. El $92 \%$ de estos estudios examinaron poblaciones de mujeres y varones adultos: un $63 \%$ se centran exclusivamente en las mujeres (ejemplos: Díaz Carrión, 2014; Rico y Gómez, 2005), mientras que el 37\% restante las analiza en comparación con los varones (ejemplos: Diéguez, Geimonde y Sinde, 2010; Sparrer, 2003). El 8\% de los estudios empíricos examinaron familias (Fuller, 2010) y consideraron a niñas, niños y adolescentes como parte de la población residente (Lugo, Alberti, Figueroa, Talavera y Monterrubio, 2011). Con respecto al origen étnico y nacionalidad, sólo uno de los estudios empíricos analiza a mujeres indígenas (Bayona, 2013). La mitad de los estudios se centra en población de América Latina y Brasil (ejemplos: Moreno y Lunar, 2006; Gabrielli, 2011); poco más de un tercio (35\%) incluye a mujeres de nacionalidad española (por ejemplo: Sarasa, 1998); con una menor representación (menos de 4\%) se encuentran los estudios que analizan a turistas europeas en Brasil (Freire-Medeiros, Nunes y Campello, 2011) o que consideran una muestra internacional de mujeres y varones (Prodanova y San Martín, 2013).

Esta síntesis de los métodos de análisis y características de las muestras de estudio, brindó un punto de partida para examinar algunas de las cuestiones epistemológicas de las investigaciones. También permitió descubrir cómo se construye el conocimiento con relación a los temas que emergieron en el análisis. A continuación se describen las generalidades de las temáticas de investigación.

\subsection{Las temáticas y marcos teóricos de investigación}

El interés de esta revisión integradora no fue cuantificar el número de artículos que abordan ciertas temáticas o emplean determinados conceptos o marcos teóricos, sino inferir algunas generalizaciones en cuanto a los temas más relevantes que han surgido en la literatura sobre turismo, género y mujeres en la región iberoamericana, así como respecto a los marcos teórico-conceptuales que se han desarrollado para abordar dichas temáticas. Las temáticas identificadas no son mutuamente excluyentes, sino que reflejan 
matices en la literatura. En los estudios analizados se observa una tendencia similar a la identificada por Gibson (2001) en la literatura anglosajona, donde los artículos suelen centrarse en uno de las tres áreas siguientes: a) las y los turistas (diferencias de género en el consumo turístico y comportamiento en los viajes); b) las anfitrionas y los anfitriones (diferencias de género en la producción turística, oportunidades de empleo, segregación laboral y discriminación por género); y c) el turismo como parte de las construcciones sociales de género (objetos e imágenes de la promoción turística identificados como femeninas o masculinas).

Entre los artículos centrados en las y los turistas, emergen temáticas como la incorporación histórica de las mujeres en los viajes y el turismo (Schlüter, 2008 y 2010); las interacciones sociales y la creación de lazos emocionales entre mujeres turistas y miembros de la población local (Freire-Madeiros, Nunes y Campello, 2011); así como el comportamiento y las preferencias diferenciadas entre mujeres y varones en el consumo de servicios o productos turísticos (Prodanova y San Martín, 2013; Rodríguez, Celemín, Rubio y Alonso, 2012; Serrano, Zarza y Serrano, 2013). Se distinguen claramente los enfoques históricos y sociológicos que se emplean para abordar las primeras temáticas (las mujeres en los viajes y el turismo, interacciones sociales entre turistas y población local); mientras que los enfoques económico-administrativos (teorías de gestión y marketing) y algunos conceptos de psicología (comportamiento, necesidades, preferencias y percepciones) son los más recurridos para el análisis del consumo turístico diferenciado entre mujeres y varones. De alguna manera, estos estudios ayudan a dar visibilidad a las mujeres en la historia del turismo y a sus necesidades específicas como turistas, aunque no siempre ahondan en las causas de esa falta de visibilidad o de escaso reconocimiento de las mujeres en las prácticas y en el consumo turístico.

En los artículos centrados en las anfitrionas y los anfitriones, surge una temática consistente que se refiere a la inserción laboral de las mujeres en el turismo, ya sea en calidad de empleadas del sector público (Rujano y Lunar, 2010) o del sector privado (Moreno y Lunar, 2006; Sarasa, 1998; Sigüenza, 2011). Surge también el interés por examinar la desigualdad laboral por género (Sigüenza, Brotons y Huete, 2013), así como la representación de las mujeres en la gestión de empresas turísticas (Tomio y Ullrich, 2011) o su participación en los niveles más altos de poder (Alonso, 2009). En estos estudios, se discuten conceptos que evidencian algunas desventajas que enfrentan las mujeres como consecuencia de los sistemas de género que se construyen socialmente y relacionan a ciertas actividades como «trabajo de mujeres». Destacan los conceptos de: trabajo doméstico, trabajo productivo, segmentación horizontal y vertical; brecha de género, techo de cristal, doble o triple jornada y conciliación laboral, entre otros. La finalidad de estos estudios es mostrar tanto los avances alcanzados, como las dificultades que siguen enfrentando las mujeres en el ámbito laboral turístico con relación a la igualdad de oportunidades y la representación paritaria en puestos de responsabilidad.

Otra temática relacionada se refiere a la participación de las mujeres en el desarrollo de iniciativas turísticas privadas o comunitarias en espacios rurales o naturales. El interés por esta temática surge a raíz de la evidente transformación de los espacios rurales, primero en el contexto europeo y posteriormente en el latinoamericano, así como del reconocimiento de las mujeres como actrices activas en el desarrollo de sus comunidades. Investigaciones 
pioneras como la de García-Ramón, Cànoves y Valdovinos (1995) abrieron la pauta para examinar el papel de ese sector en el desarrollo rural, particularmente en el contexto de la promoción del turismo como opción productiva en el proceso de reestructuración económica de las zonas rurales de Europa y de España. Al centrarse en el papel de las mujeres, los estudios suelen matizar las oportunidades brindadas por el turismo rural, puesto que contrastan tanto los efectos positivos como los negativos que tiene dicha actividad sobre las relaciones de género y sobre el trabajo doméstico y productivo de dicho sector (Sparrer, 2003; Díaz Carrión, 2010).

Por lo general, estos estudios tienen un mayor tratamiento teórico-conceptual e incorporan enfoques antropológicos, sociológicos, de desarrollo local, de la geografía de género, teorías feministas y perspectiva de género. Las mujeres no son homogéneas; al contrario, destaca la diversidad de poblaciones examinadas: mujeres rurales (por ejemplo: Rico y Gómez, 2005; Fernández y Martínez, 2010), indígenas (Bayona, 2013), urbanas que se desplazan a las zonas rurales para trabajar en iniciativas de ecoturismo o turismo de aventura (Díaz Carrión, 2010 y 2012). Para enriquecer el análisis se suelen incorporar otras categorías que interactúan con el género, como la clase, el origen étnico, la procedencia o los niveles de competencia. Una relación que emerge en estos estudios es la de turismo y «empoderamiento» de las mujeres, principalmente debido a que las iniciativas que se analizan son lideradas por ellas, ya sea en empresas privadas (Fernández y Martínez, 2010) o empresas comunitarias (ver Díaz Carrión, 2010 y 2012). En la sección de discusión se abordarán las implicaciones de incorporar el concepto de empoderamiento en los estudios sobre turismo, género y mujeres.

Otros temas emergentes se refieren a la transformación de las relaciones familiares y de género entre los grupos que participan en el desarrollo turístico de sus comunidades (por ejemplo: Fuller, 2010); el papel de las mujeres indígenas como productoras y vendedoras de artesanías de consumo turístico (Bayona, 2013) y el papel de las mujeres en el comercio informal en destinos turísticos (Gámez, Ivanova y Wilson, 2011).

Finalmente, en los estudios centrados en el turismo como parte de las construcciones sociales de género, destaca el tema de la sexualización de la mujer desde las imágenes empleadas en la promoción turística de un destino (Siqueira y Siqueira, 2009; Norrild, 2007) o desde el imaginario social y los discursos turísticos dominantes (Gomes, 2013). También se habla de la sexualización de la mujer en los artículos que examinan las modalidades de turismo relacionadas con elementos sexuales (Norrild, 2007), o las prácticas del turismo sexual (Gabrielli, 2011). Estos artículos construyen sus marcos de referencia a partir de teorías sociales críticas y en menor medida, de las teorías feministas. Por ejemplo, retoman y discuten las aportaciones de Pierre Bourdieu sobre la dominación masculina y la violencia simbólica contra las mujeres; los conceptos de poder y saber de Michel Foucault; o el paradigma de la «tercera mujer» de Gilles Lipovetsky. Además, incorporan conceptos como los estereotipos de género, el cuerpo femenino, la erotización de las mujeres, el discurso hegemónico y las relaciones de poder entre los géneros. También se aprecia la compleja interrelación entre categorías de análisis: cuerpo, cultura, género, origen étnico, poder, raza, entre otras. Los análisis abarcan tanto las dimensiones culturales, como las políticas. Una de sus principales aportaciones es evidenciar los discursos e imaginarios hegemónicos en la promoción turística. 


\section{CONCLUSIONES}

Aunque esta revisión estuvo centrada exclusivamente en artículos científicos publicados en revistas indizadas de turismo y excluye otras fuentes importantes (libros o ponencias presentadas en congresos), ofrece un primer diagnóstico del estado actual de la investigación sobre turismo, género y mujeres en la región iberoamericana. Se reconoce que en otras revistas especializadas en Geografía, Ciencias Sociales y Antropología se pueden encontrar artículos sobre la temática tratada, pero no fueron incluidos en esta revisión. Si bien en los países de habla inglesa es posible argumentar sobre una mayor complejidad y diversidad en las investigaciones sobre turismo, género y mujeres (Swain, 2005), la presente revisión muestra que en el contexto iberoamericano todavía quedan muchos temas por explicar con relación a las dinámicas de género y las cuestiones de poder entre mujeres y varones tanto en las prácticas turísticas, como en las iniciativas de desarrollo turístico.

Algunas revistas se centran más en las dimensiones sociales y culturales del turismo y por ende, dan mayor cabida a los análisis de género (Cuadernos de Turismo, PASOS, Estudios y Perspectivas en Turismo, Revista Brasileira de Pesquisa em Turismo), mientras que otras muestran un enfoque más práctico (Análisis Turístico, Estudios Turísticos, Encontros Turísticos Tourism\& Management Studies), o especializado (TURyDES), además de presentar un menor número de artículos que incorporan el análisis de las dimensiones de género. Estos resultados sugieren que las temáticas de género y participación de las mujeres en el turismo aún no tienen una presencia consolidada en la investigación producida en la región iberoamericana. Además, tres cuartas partes de los artículos analizados en la muestra se publicaron en los últimos cuatro años, por lo que se espera que el interés en los temas de turismo y género siga creciendo en el futuro próximo.

La síntesis de los métodos empleados en las investigaciones arroja una marcada preferencia por los análisis cualitativos, en particular por las entrevistas semiestructuradas y las entrevistas en profundidad. Lo anterior probablemente responda a la necesidad de examinar las formas en que las mujeres experimentan su participación en el turismo, bien como turistas, empleadas, gestoras o, como propietarias y socias de iniciativas turísticas privadas y comunitarias. En este sentido, los métodos cualitativos emergen como los más apropiados para visibilizar el trabajo de las mujeres y dar voz a sus experiencias subjetivas (ver Díaz Carrión, 2010, 2012 y 2014).

En segundo lugar se encuentran los métodos cuantitativos y mixtos. Si bien la mayoría de los estudios que emplean métodos cuantitativos como análisis de bases de datos, encuestas o cuestionarios ad hoc parten de un enfoque económico-administrativo, no se puede ignorar el potencial de estos métodos para revelar patrones y situar la participación de las mujeres así como las desigualdades que enfrentan en ámbitos como la educación formal, la investigación científica y el empleo, entre otros. Precisamente, las investigadoras y los investigadores que incorporan métodos mixtos en la muestra analizada, justifican su elección con base en la complementariedad de los datos cuantitativos y los cualitativos y señalan la importancia de construir modelos interpretativos que permitan contrastar los datos con la teoría. 
La mayoría de los estudios son de tipo empírico y sólo el $12 \%$ responde a análisis de tipo histórico o teórico-conceptual. Entre estos últimos no se encontró ningún artículo de revisión de la literatura. La escasa presencia de artículos teóricos tendría sentido en un contexto de madurez en la investigación sobre turismo y género (como ocurre en los países de habla inglesa), pero en la región iberoamericana representa en realidad un área de oportunidad. Si se quiere consolidar la línea de investigación sobre turismo, género y mujeres en los países de la región, es indispensable promover los debates teóricos, conceptuales y metodológicos, que permitan reexaminar temas ya establecidos en las agendas locales, nacionales o regionales, así como plantear nuevas preguntas de investigación.

Con relación a la autoría, el grueso de la investigación en este campo la están realizando mujeres, quienes por lo general estudian el fenómeno turístico en sus países de origen (España, México, Brasil, Argentina, Venezuela o Perú) y en el menor de los casos, analizan el desarrollo turístico en otros destinos (ejemplo: Bayona, 2013; Norrild, 2007). En su mayoría las autoras y autores no se definen como feministas, pero sí emplean perspectivas feministas (por ejemplo: Díaz Carrión, 2010;Gabrielli, 2011; Gomes, 2013;Norrild, 2007), o recurren a la perspectiva de género como herramienta de análisis (Alonso, 2009; Fernández y Martínez, 2010; Gamez, Invanova y Wilson, 2011; Sigüenza, 2011).

Entre los estudios analizados se observa un creciente interés por abordar las desigualdades de género que en pleno siglo XXI deberían estar superadas, pero que persisten a razón de la fortaleza de las construcciones socio-culturales que asignan a las mujeres los espacios privados y el trabajo reproductivo no remunerado, mientras se espera que los hombres se desenvuelvan en los espacios públicos y en el trabajo productivo remunerado (Sparrer, 2003).

En países como España, donde no sólo se ha alcanzado la igualdad de género en el ámbito educativo, sino que incluso las mujeres se encuentran sobrerrepresentadas en los programas de estudios superiores en turismo (Iñiguez, Plumed y Latorre, 2013), las investigaciones concluyen que se dista mucho de alcanzar la igualdad efectiva entre mujeres y varones en el mercado laboral (ver Sarasa, 1998; Sigüenza, 2011; Sigüenza, Brotons y Huete, 2013). Particularmente, las mujeres siguen enfrentando serias dificultades para insertarse en los niveles de mayor responsabilidad en los sectores público, privado, de investigación e innovación. Como señala Sigüenza (2011:108): «La asunción de las responsabilidades familiares por parte de la mujer es la principal causa que la mantiene alejada de los puestos directivos.»

En los países de América Latina, además del análisis de la participación de las mujeres en el mercado laboral turístico (Moreno y Lunar, 2006) y los impactos diferenciados del desarrollo turístico sobre mujeres y varones (Díaz Carrión, 2010); se detecta un creciente interés por examinar las estrategias que desarrollan las mujeres para compatibilizar su trabajo en el turismo en los sectores formal e informal, con sus responsabilidades familiares (ver Díaz Carrión, 2014; Tomio y Ullrich, 2011; Fernández y Martínez, 2010; Fuller, 2010). Estos estudios buscan visibilizar las experiencias cotidianas de las mujeres y contribuyen a la construcción de categorías de análisis cada vez más socorridas en la investigación sobre turismo, género y mujeres (representaciones, vida cotidiana, conciliación laboral, gestión de espacios y tiempos, entre otras). 
Finalmente, un concepto que comienza a retomarse desde las teorías feministas y los estudios de género y desarrollo en los estudios analizados es el de empoderamiento. Por lo general, la incorporación del empoderamiento responde al interés de analizar si el turismo contribuye o no a mejorar las condiciones de vida de las mujeres que participan en su producción y/o consumo.

Como se ha observado en esta revisión integradora, los estudios sobre turismo, género y mujeres en la región iberoamericana se encuentran en una fase inicial de producción académica, pero tienen el potencial de identificar desigualdades de género e impactos diferenciados del desarrollo turístico, así como visibilizar las aportaciones, experiencias e intereses de las mujeres como agentes activas en el consumo y la producción turística. Una oportunidad importante es la de enriquecer los debates teóricos, conceptuales y metodológicos que contribuyan a la incorporación de la perspectiva de género en los estudios turísticos. Además, se requiere realizar colaboraciones interinstitucionales para contrastar las problemáticas de género en diferentes contextos socioculturales e identificar estrategias que contribuyan a los objetivos de la igualdad de oportunidades y el empoderamiento de las mujeres en el turismo.

\section{BIBLIOGRAFÍA}

ALONSO, M.M. (2009): «Los consejos de administración de las cadenas hoteleras mundiales. Análisis desde la perspectiva de género», en Estudios y Perspectivas en Turismo, vol. 18, n 2, pp. 112-125. Disponible en: http://www.estudiosenturismo.com. ar/PDF/V18/v18n2a1.pdf

BAYONA, E. (2013): «Textiles para turistas: tejedoras y comerciantes en los Altos de Chiapas», en PASOS. Revista de Turismo y Patrimonio Cultural, vol. 11, no 2, pp. 371-386. Disponible en: http://www.pasosonline.org/Publicados/11213/PS0213_08.pdf

COOPER, H. (1982): «Scientific guidelines for conducting integrative research reviews», en Review of Educational Research, vol. 52, pp. 291-302.

DÍAZ CARRIÓN, I.A. (2010): «Ecoturismo comunitario y género en la reserva de la biosfera de Los Tuxtlas (México)», en PASOS. Revista de Turismo y Patrimonio Cultural, vol. 8, no 1, pp. 151-165. Disponible en: http://www.pasosonline.org/ Publicados/8110/PS0110_12.pdf

DÍAZ CARRIÓN, I.A. (2012): «Turismo de aventura y participación de las mujeres en Jalcomulco (México)», en PASOS. Revista de Turismo y Patrimonio Cultural, vol. 10, no 5, pp. 531-542. Disponible en: http://www.pasosonline.org/Publicados/10512/ PS0512_08.pdf

DÍAZ CARRIÓN, I.A. (2014): «Ecoturismo y vida cotidiana de las mujeres en Sontecomapan (Veracruz, México)», en Cuadernos de Turismo, no 34, pp. 69-88. Disponible en: http:// revistas.um.es/turismo/article/view/203031/164221

DIÉGUEZ, M. I., GUEIMONDE, A. y SINDE, A.I. (2010): «Éxito empresarial y género en el turismo rural», en Encontros Científicos-Tourism \& Management Studies [on line], no 6, pp. 82-93. Disponible en: http://www.scielo.gpeari.mctes.pt/pdf/ec/n6/ n6a09.pdf 
DIÉGUEZ, M.I., GEUIMONDE, A. y BLANCO, L. (2011): «Gap tecnológico de género en turismo rural», en Análisis Turístico, no 11, pp. 63-68.

ENLOE, C. (1989): Bananas. Beaches and Bases: Making Feminist Sense of International Politics, Berkeley, University of California Press.

FERNÁNDEZ, M.J. y MARTÍNEZ, L.A. (2010): «Participación de las mujeres en las empresas turísticas privadas y comunitarias de Bahías de Huatulco, México. ¿Hacia un cambio en el rol de género?», en Cuadernos de Turismo, n 26, pp. 129-151. Disponible en: http://revistas.um.es/turismo/article/view/116311/110071

FREIRE-MEDEIROS, B., NUNES, F. y CAMPELLO, L. (2011): «Sobre afetos e fotos: Volunturistas em uma favela carioca», en Revista Brasileira de Pesquisa em Turismo, vol. 5, no 2, pp. 157-176. Disponible en: http://www.rbtur.org.br/rbtur/article/ view/424/474

FULLER, N. (2010): «Lunahuaná, un destino turístico. Transformaciones en la composición social, economía familiar y relaciones de género», en PASOS. Revista de Turismo y Patrimonio Cultural, vol. 8, n 2, pp. 293-304.Disponible en: http://www.pasosonline. org/Publicados/8210/PS0210_04.pdf

GABRIELLI, C. (2011): «Intersecções entre o mercado turístico e o mercado do sexo em Salvador, Bahía, Brasil», en Revista Brasileira de Pesquisa em Turismo, vol. 5, n 2 , pp. 136-156. Disponible en: http://www.rbtur.org.br/rbtur/article/view/377/454

GÁMEZ, A.E., IVANOVA, A. y WILSON, T.D. (2011): «Género y comercio informal en destinos turísticos. El caso de las vendedoras de playa en Los Cabos, Baja California Sur, México», en TURyDES. Revista de investigación en turismo y desarrollo local, vol. 4, no 9, pp. 1-31. Disponible en: http://www.eumed.net/rev/turydes/09/giw.pdf

GARCIA-RAMON, M.D., CANOVES, G., VALDOVINOS, N. (1995): «Farm tourism, gender and environment in Spain», en Annals of Tourism Research, vol. 22, n 2, pp. 267-282.

GIBSON, H.J. (2001): «Gender in tourism: Theoretical perspectives» en Women as producers and consumers of tourism in developing regions, Westport, Connecticut, Praeger, pp. 19-43.

GOMES, M.S. (2013): «[Des][Re]construcciones del imaginario social <mujer Brasileña> en los discursos turísticos sobre Brasil en Portugal», en Estudios y Perspectivas en Turismo, vol. 22, no 2, pp. 216-234. Disponible en: http://www.estudiosenturismo.com. ar/PDF/V22/N02/v22n2a03.doc.pdf

HARDING, S. (1986): The science question in feminism. New York, Cornell University Press. HENDERSON, K.A. (1994): «Perspectives on analyzing gender, women, and leisure», en Journal of Leisure Research, vol. 26, n 2, pp. 119-137.

HENDERSON, K.A. (1996): «One size doesn't fit all: The meanings of women's leisure», en Journal of Leisure Research, vol. 28, n 3, pp. 139-154.

HENDERSON, K.A. y GIBSON, H.J. (2013): «An integrative review of women, gender, and leisure: Increasing complexities», en Journal of Leisure Research, vol. 39, n 2 , pp. $115-135$.

HENDERSON, K.A. y HICKERSON, B.D. (2007):«Women and leisure: Premises and performances uncovered in an integrated review», en Journal of Leisure Research, vol. $45, \mathrm{n}^{\circ} 4$, pp. $115-135$. 
IÑIGUEZ, T., PLUMED, M. y LATORRE, M.P. (2013): «Tourism education and research in Spain from a gender perspective», en Rotur. Revista de Ocio y Turismo, n 6, pp. 51-65. Disponible en: http://www.rotur.es

KINNAIRD, V. y HALL, D. (1994):Tourism: A Gender Analysis. Chichester, John Wiley \& Sons.

KINNAIRD, V. y HALL, D. (1996): «Understanding tourism processes: a gender-aware framework», en Tourism Management, vol. 17, n 2, pp. 96-102.

LUGO, G., ALBERTI, M.P., FIGUEROA, O.L., TALAVERA, D. y MONTERRUBIO, J.C. (2011): «Patrimonio cultural y género como estrategia de desarrollo en Tepetlaoxtoc, Estado de México», en PASOS. Revista de Turismo y Patrimonio Cultural, vol. 9, n ${ }^{\circ}$ 4, pp. 599-612. Disponible en: http://www.pasosonline.org/Publicados/9411/PS0411_09.pdf

MORENO, F.F. y LUNAR, R.A. (2006): «Turismo y género: Empleo de la mujer en la actividad turística en la Isla Margarita, Estado Nueva Esparta», en PASOS. Revista de Turismo y Patrimonio Cultural, vol. 4, no 3, pp. 373-389. Disponible en: http://www. pasosonline.org/Publicados/4306/PS060306.pdf

NORRILD, J. (2007): «Relación entre turismo, género y sexo. El caso de Buzios - Brasil», en PASOS. Revista de Turismo y Patrimonio Cultural, vol. 5, n 3, pp. 331-341. Disponible en: http://www.pasosonline.org/Publicados/5307/PS050307.pdf

NORRILD, J. (2010): «Rouge y tacos al volante. La relación entre autos y mujeres en el ámbito turístico de Argentina», en Estudios y Perspectivas en Turismo, vol. 19, pp. 471-499. Disponible en:

http://www.estudiosenturismo.com.ar/PDF/V19/v19n4a2.pdf

PRITCHARD, A., MORGAN, N., y ATELJEVIC, I. (2011): «Hopeful tourism: A new transformative perspective», en Annals of Tourism Research, vol. 38, pp. 941-963.

PRODANOVA, J. y SAN MARTÍN, S. (2013): «Estudio sobre el impacto de género y las emociones en el comportamiento de compra online de viajes», en Análisis Turístico, $\mathrm{n}^{\circ} 13$, pp. 1-12.

RICO, M. y GÓMEZ, M.J. (2005): «La participación empresarial de la mujer en las iniciativas de turismo rural en Castilla y León», en Estudios Turísticos, n 166, pp. 97-113.

RODRÍGUEZ, J.M., CELEMÍN, M.S., RUBIO, L. y ALONSO, M.M. (2012): «Preferencias diferenciales de los hombres y mujeres como clientes de hoteles. Una aplicación empírica en una universidad Madrileña», en Cuadernos de Turismo, no 29, pp. 231245. Disponible en:

http://revistas.um.es/turismo/article/view/153861/135561

RUJANO, M. y LUNAR, R.A. (2010): «Turismo y género: desempeño laboral de la mujer en los organismos turísticos públicos del estado Nueva Esparta», en PASOS. Revista de Turismo y Patrimonio Cultural, vol. 8, nº1, pp. 195-210. Disponible en: http://www.pasosonline.org/Publicados/8110/PS0110_15.pdf

SARASA, J.L.A. (1998): «Turismo y empleo femenino», en Cuadernos de Turismo, no 1 , pp. 9-27. Disponible en: http://revistas.um.es/turismo/article/view/24591/23911

SCHLÜTER, R. (2008): «Mujer y turismo. Vestimenta e interacción social en los centros turísticos de Argentina durante la década de 1930», en Cuadernos de Turismo, $\mathrm{n}^{\circ} 21$, pp. 181-199. Disponible en: http://revistas.um.es/turismo/article/view/25041/24331 
SCHLÜTER, R. (2010): «Feminity at thebeach - issuesonfashion, gender and tourism in Argentina», en Revista Brasileira de Pesquisa em Turismo, vol. 4, no 3, pp. 71-84. Disponible en: http://www.rbtur.org.br/rbtur/article/view/340/381

SERRANO, H. P., ZARZA, M.P. y SERRANO, C. (2013): «Turismo cultural, transiciones en términos de género y su prospectiva», en El Periplo Sustentable, n 25, pp. 135-158. Disponible en: http://www.uaemex.mx/plin/psus/articulo_06.pdf

SIGÜENZA, M.C. (2011): «La mujer en el subsector del alojamiento. El caso de la Costa Blanca (Alicante, España)», en Investigaciones Turísticas, n 2, pp. 102-119. Disponible en: http://rua.ua.es/dspace/bitstream/10045/20080/1/InvestigacionesTuristicas_02_05.pdf

SIGÜENZA, M.C., BROTONS, M. y HUETE, R. (2013): «The evolution of gender inequality in tourism employment in Spain», en Rotur. Revista de Ocio y Turismo, $\mathrm{n}^{\circ}$ 6, pp. 182-200. Disponible en: http://www.rotur.es

SIQUEIRA, E.D. y SIQUEIRA, D. (2009): «El cuerpo femenino. Comunicación, poder y turismo», en Estudios y Perspectivas en Turismo, vol. 18, pp. 193-207.Disponible en: http://www.estudiosenturismo.com.ar/PDF/V18/v18n2a6.pdf

SPARRER, M. (2003): «Género y turismo rural. El ejemplo de la costa coruñesa», en Cuadernos de Turismo, no 11, pp.181-197. Disponible en: http://revistas.um.es/turismo/ article/view/19441/18801

STANLEY, L. y WISE, S. (1993):Breaking out again. Londres, Routledge.

SWAIN, M. B. (1995): «Gender in tourism», en Annals of Tourism Research, vol. 22, pp. 247-266.

SWAIN, M.B. (2005): «Las dimensiones de género en la investigación sobre turismo: Temas globales, perspectivas locales», en Política y Sociedad, vol. 42, pp. 25-37.

TOMIO, M. y ULLRICH, D.R. (2011): «Gestión de las empresas turísticas. La representación de las mujeres en Bluenau - SC, Brasil», en Estudios y Perspectivas en Turismo, vol. 20, pp. 425-440. Disponible en: http://www.estudiosenturismo.com. ar/PDF/V20/N02/v20n2a09.pdf

ZAHO, W. y RITCHIE, J.R. (2007): «An investigation of academic leadership in tourism research: 1985-2004», en Tourism Management, vol. 28, n 2, pp. 476-490. 
